\title{
Commutative Rings of Partial Differential Operators and Lie Algebras
}

\author{
O. A. Chalykh and A.P. Veselov \\ Moscow State University, SU 117234 Moscow, USSR
}

\begin{abstract}
We give examples of finite gap Schrödinger operators in the twodimensional case.
\end{abstract}

The problem of characterizing rings of commuting ordinary differential operators (ODO) was introduced and investigated by Burchnal and Chaundy [1]. Modern investigations begin with the work of Novikov [2], who noticed that a Schrödinger operator with periodic potential $L=-d^{2} / d x^{2}+u(x)$, which has a commuting ODO of odd order, possesses "good spectral properties," namely: its spectrum has only a finite number of gaps (see also [3,4]). Subsequently, Krichever [5] completed the classification of all commutative rings of ODO with scalar coefficients. The generalization of this classification on the case of matrix coefficients was made by Grinevich [6].

The attempts to understand the many-dimensional case, for instance, the case of the two-dimensional Schrödinger operator $L$, lead B.A. Dubrovin, I.M. Krichever, and S.P. Novikov to the formulation of a new problem [7, 8]. Now one wants to characterize the operators $L$, which are ,finite-gap on one energy level." Such operators can be included in the rings of differential operators, commutative $\bmod L:[L, A]=B L$ (see [7]). In the general case the change of energy level violates the finite-gap property. This paper presents in particular the attempt to answer the question posed by S.P. Novikov in 1976 about the operators $L$, which are finitegap in every energy level, and the corresponding deformations of curves and divisors. Up to now actually only the separable case $L=\partial_{x}^{2}+\partial_{y}^{2}+u(x)+v(y)$ with $u(x)$ and $v(y)$ being the finite gap potentials, was known where this phenomenon occurred. We will show here that there are new examples of this type and conjecture that there are no other operators with analogous properties.

We say that the commutative ring $\mathscr{R}$ of differential operators in $\mathbb{R}^{n}$ is complete if there exist $n$ operators $L_{i}\left(x, \frac{\partial}{\partial x}\right) \in \mathscr{R}(i=1, \ldots, n)$ with algebraically independent highest symbols $P_{i}(k)$. A complete commutative ring $\mathscr{R}$ is called a supercomplete if $\mathscr{R}$ is not contained in any commutative ring, which is generated by 
only $n$ operators. For $n=1$ all supercomplete commutative rings are described by the Burchnal-Chaundy-Krichever theory.

Problem. Describe all supercomplete commutative rings of differential operators in $\mathbb{R}^{n}$, containing some Schrödinger operator $L=-\Delta+u(x)$.

In other words we want the corresponding quantum problem with $H=p^{2}+u(q)$ to have at least $(n+1)$ commuting integrals instead of the usual $n$ integrals. The precise formulation of the last property is due to I.M. Krichever, who called such operators algebraic [9].

Now we describe some Schrödinger operators, which are conjectured to have this property.

Let $G$ be a simple complex Lie algebra of rank $n, R$ be its root system in $\mathbb{R}^{n}, R_{+}$ be the set of positive roots, the brackets denote some scalar product in $\mathbb{R}^{n}$, invariant under the action of Weyl group $W$ (see [10]).

Consider the following Schrödinger operator

1. $H_{G}=-\Delta+\sum_{\alpha \in R_{+}} g_{\alpha} \mathscr{P}((\alpha, x))$, where $\mathscr{P}(z)$ is Weierstrass elliptic function, and its degenerations;

2. $H_{G}=-\Delta+\sum_{\alpha \in R_{+}} g_{\alpha} \omega^{2} \sin ^{-2} \omega(\alpha, x)$;

2'. $H_{G}=-\Delta+\sum_{\alpha \in R_{+}} g_{\alpha} \omega^{2} \sin h^{-2} \omega(\alpha, x)$;

3. $H_{G}=-\Delta+\sum_{\alpha \in R_{+}} g_{\alpha}(\alpha, x)^{-2}$.

When $G=A_{n}$ such quantum systems for (3) were considered by Calogero [11], in the trigonometric case (2) it was considered by Sutherland [12]. After the paper of Moser [13] where Lax representation for the corresponding classical systems was found, F. Calogero proposed the generalization of Moser's matrix, which led to solving some functional equation [14]. The general solution of that equation is expressed in elliptic function and leads to the potential $\mathscr{P}(x)^{1}$ M.A. Olshanetsky and A.M. Perelomov were the first to propose a generalization using the other root systems [15,16] (see also the review [17]). They succeeded in proving in some cases the complete integrability of these systems, in the sense of the existence of $n$ commutative integrals $I_{1} \ldots I_{n}$.

The main goal of this paper is to give some motivation for the following conjectures, belonging to one of the authors (A.P.V.).

Conjecture 1. If $g_{\alpha}$ in (1)-(3) has a form $g_{\alpha}=m(m+1)(\alpha, \alpha), m \in Z$, then the rings of all quantum integrals of this problem are supercomplete.

Conjecture 2. There are no other supercomplete and nonseparable commutative rings of differential operators, containing some Schrödinger operator $H=-\Delta+u(x), x \in \mathbb{R}^{n}, n \geqq 2$.

\footnotetext{
${ }^{1}$ It was proven approximately simultaneously and independently by $\mathrm{H}$. Airault, F. Calogero, M. Olshanetsky and A. Perelomov, A. Stepin, and S. Pidkuiko
} 
We call such a ring separable if it contains an operator in a smaller number of variables. For example, the ring of quantum integrals of the Calogero system is separable, because it contains the operator

$$
L_{0}=\sum_{i=1}^{n} \partial_{i}
$$

but after reducing we come to a nonseparable ring on the hyperplane

$$
\sum_{i=1}^{n} x_{i}=0
$$

In this paper we will prove conjecture 1 in some special cases: case (3) for all Lie algebras of rank 2 and for the series $A_{n}$; case (2), (2') for Lie algebras or rank 2. Concerning conjecture 2 we have only some intuition, which we will present now.

\section{Motivations: Commutative Mappings and Lie Algebras}

Here we follow the papers of one of the authors $[18,19]$. Let's consider another problem, which seems to be simpler. Let $P(z)$ be a polynomial $P(z)=a_{n} z^{n}+\ldots+a_{0}$. It determines the mapping $z \rightarrow P(z)$.

Problem. Describe all $P(z)$, such that there exists commuting polynomial mapping $z \rightarrow Q(z): P \circ Q=Q \circ P$ and the sets of iterations of $P$ and $Q$ do not intersect.

This problem was solved independently and almost simultaneously by Julia [20], Fatou [21], and Ritt [22]. The proof turned out to be complicated, but the answer very simple: every such mapping up to linear transformations and change of sign must be determined by the Tchebycheff polynomials $T_{k}(z)=\cos k \arccos z$ or by the formula $z \rightarrow z^{k}$.

For example, when $P(z)=z^{2}+c$, the commuting polynomial without common iterates will exist only in two cases: $c=0$ or $c=-2$. The commuting polynomial has a form $Q=z^{3}$ or correspondingly $Q=z^{3}-3 z$. In 1985 one of the authors discovered the unexpected deep analogy between this problem and the problem about commuting differential operators (see $[18,19])$. It is very natural to hope for such analogy in the many-dimensional case and to see the commuting polynomial mappings $\mathbb{R}^{n} \rightarrow \mathbb{R}^{n}$ or $\mathbb{C}^{n} \rightarrow \mathbb{C}^{n}$. In the paper [18] the following construction of such mappings, which generalize the construction of Tchebycheff mappings and connected with Lie algebras, was proposed.

Let $G$ be a simple complex Lie algebra of rank $n, H$ its Cartan subalgebra, $H^{*}$ its dual space, $\mathscr{L}$ a lattice of weights in $H^{*}$, generated by the fundamental weights $\omega_{1}, \ldots, \omega_{n}, L$ the dual lattice in $H$ (see [10]). Let's consider the mapping

$$
\mathbb{Q}_{G}: H / L \rightarrow \mathbb{C}^{n,} \mathbb{Q}_{G}=\left(\phi_{1}, \ldots, \phi_{n}\right), \phi_{k}=\sum_{w \in W} \exp \left(2 \pi i w\left(\omega_{k}\right)\right),
$$

where $W$ is the Weyl group, acting on the space $H^{*}$. According to the Chevalley theorem [10], the algebra of exponential invariants of $W$ is freely generated by 
$\phi_{1}, \ldots, \phi_{n}$. Therefore there exist the polynomial mappings $P_{G}^{k}$, which are determined by the relation

$$
\mathbb{Q}_{G}(k x)=P_{G}^{k}\left(\mathbb{Q}_{G}(x)\right) \text {. }
$$

From the definition $P_{G}^{k} \circ P_{G}^{l}=P_{G}^{k l}=P_{G}^{l} \circ P_{G}^{k}$.

Dynamical properties of this mapping were investigated by the authors in $[18,19,22,24]$. In particular, it was proven that nonisomorphic Lie algebras determine nonequivalent commutative families of mappings $P_{G}^{k}$.

If we consider the abelian variety $M_{G}=\mathbb{C}^{n} / L+\tau L$, which is isomorphic to the product of elliptic curves with parameter $\tau(\operatorname{Im} \tau>0, \tau \in \mathbb{C})$, then one can use the corresponding analogue of Chevalley's theorem, proved by Looijenga [25], Bernstein and Schwarzman [26]. According to this theorem the quotient of this variety by the natural action of the Weyl group $W$ is the weighted projective space $\mathbb{C} P^{n}$. Using this theorem one can define the commuting mapping $\mathbb{C} P^{n} \rightarrow \mathbb{C} P^{n}$, corresponding to any endomorphism $M_{G}$, for example $Z \rightarrow m Z$.

This construction [18] can be considered as the generalization of a result of Ritt [22], who proved that all rational mappings $f: \mathbb{C} P^{1} \rightarrow \mathbb{C} P^{1}$, which possess the appropriate commuting mappings, must be the "multiplication law" for some elliptic functions or its degenerations (see [22]).

It can be shown that all similar generalizations lead to the root systems.

The following step is to understand which commutative rings of differential operators can be considered as the analogue of our mappings. Attempting to find such quantum systems, one of the authors came to the previous conjectures. The quantum Toda lattice and its generalization found by Bogoyavlensky [27] and also connected with Lie algebras do not fit for our purpose. It follows from the consideration of the simplest case of two particles, because the potential $u=\exp x$ is not finite-gap. If we try to find the integrable quantum system with finite-gap potential, we will come to the systems $(1-3)$.

Notice that the operator (1) with $g_{\alpha}=(\alpha, \alpha) m(m+1)$ can be considered as the generalization of the well-known Lamé operator. ${ }^{2}$

\section{The Analytic Properties of the Eigenfunction $\psi$ of the Operators $H_{G}$ for $g_{\alpha}=m(m+1)(\alpha, \alpha)$}

We begin with the rational case:

$$
H_{G}^{m}=-\Delta+m(m+1) \sum_{\alpha \in R_{+}}(\alpha, \alpha)(\alpha, x)^{-2} .
$$

Following [28], let us consider the function $\psi(k, x), k, x \in R^{n}\left(\mathbb{C}^{n}\right)$, of the form

$$
\psi=P(k, x) \exp (k, x),
$$

where $P(k, x)$ is a polynomial in $k$ with the highest term $A(k)=\prod_{\alpha \in R_{+}}(\alpha, k)$.

\footnotetext{
${ }^{2}$ One should observe that all these operators are singular on the reals. Only for $A_{1}$, i.e. in onedimensional case is it possible to make the potential $m(m+1) \mathscr{P}(x)$ nonsingular by a half-period shift
} 
Theorem 1. Let the function $\psi$ be of the form (5) and have the following property:

$$
\left(\alpha, \frac{\partial}{\partial k}\right) \psi=0
$$

when $(\alpha, k)=0$ for all $\alpha \in R_{+}$. Then $\psi$ is the eigenfunction of a supercomplete commutative ring, which contains the operator (4) for $m=1$ and is isomorphic to the ring $K$ of all polynomials $f(k)$ with the property $(6)$. This isomorphism is given by the formula $f \rightarrow L_{f}$,

$$
L_{f} \psi=f(k) \psi \text {. }
$$

Proof. The following lemma will be very useful.

Lemma. If the function $\phi=Q(k, x) \exp (k, x)$, where $Q$ is a polynomial in $k$, satisfies condition (6), then

1. the highest term of $Q$ is divisible by $A(k)$;

2. there exists a differential operator $L\left(x, \frac{\partial}{\partial x}\right)$, such that $\phi=L \psi$ under the assumption that the function $\psi$ with prescribed properties does exist.

Proof of the lemma. The first statement is evident, because the highest term must be zero on all hyperplanes $(\alpha, k)=0$ as follows immediately from (6). Notice that the uniqueness of the $\psi$-function now follows. Consider the highest term of $Q: Q=Q_{N}+\ldots, \quad Q_{N}=B(k, x) A(k)$ and the differential operator $L_{N}=B\left(\frac{\partial}{\partial x}, x\right)$. It's easy to see that the function $\phi_{N}=L_{N} \psi$ has the same highest term $Q_{N}$ and also satisfies condition (6). Consider the difference $\phi=\left(\phi-L_{N} \psi\right)$ and repeat the same procedure to prove the lemma.

In order to deduce the theorem from the lemma it is sufficient to notice that the functions $\phi=f(k) \psi$ with $f \in K$ satisfy the condition (6).

Notice that the procedure of finding the operators $L_{f}(x, \partial / \partial x)$ is absolutely effective if the explicit formula for $\psi$ is given. For example, every $W$-invariant polynomial satisfies the condition (6), therefore one can find the operators $L_{i}(x, \partial / \partial x)$, corresponding to the generators $P_{i}(k), i=1, \ldots, n$ of the ring of $W$-invariant polynomials.

It's easy to calculate from (6) the second term of $P(k, x)$ in (5):

$$
P(k, x)=A(k)-\sum_{\alpha \in R_{+}}(\alpha, \alpha)(\alpha, x)^{-1} \prod_{\beta \in R_{+} \backslash \alpha}(\beta, k)+\ldots .
$$

Using this fact one can find the operator, corresponding to

$$
P_{1}(k)=-k^{2}: L_{1}=-\Delta-2 \sum_{\alpha \in R_{+}}(\alpha, \alpha)(\alpha, x)^{-2}, L_{1} \psi=-k^{2} \psi .
$$

Notice that the completeness of our ring now follows. It's easy to check that

$$
P_{n+1}(k)=Q(k) \prod_{\alpha \in R_{+}}(\alpha, k)^{2}
$$

for every polynomial $Q(k)$ satisfies the condition (6), but is generally not expressible as a polynomial in $P_{1}(k), \ldots, P_{n}(k)$ because it is is not invariant under 
the Weyl group. So the commutative ring in our case is much richer than in the general case, where only the operators $L_{i}(x, \partial / \partial x)$ can be constructed (see $\left.[15,16]\right)$.

Let's now consider the function $\psi_{m}(k, x)$ of the form

$$
\psi_{m}(k, x)=\left(A^{m}(k)+\text { lower term }\right) \exp (k, x)
$$

and demand that $\psi_{m}$ satisfies the following condition:

$$
\left(\alpha, \frac{\partial}{\partial k}\right)\left[(\alpha, k)^{-1}\left(\alpha, \frac{\partial}{\partial k}\right)\right]^{S} \psi_{m}=0
$$

when $(\alpha, k)=0$ for all $\alpha \in R_{+}$and $s=0,1, \ldots,(m-1)$.

By similar methods one can prove that only one such function can exist and it must be the eigenfunction of supercomplete commutative ring which contains the operator (4) and is isomorphic to the ring of polynomials with the property (8).

Trigonometric Case. Let the function $\psi(k, x)$ have the form

$$
\psi_{m}(k, x)=(A(k)+\text { lower terms }) \exp (k, x),
$$

but instead of (6) demand that relation

$$
\psi(k+i \omega \alpha, x) \equiv \psi(k-i \omega \alpha, x), \quad i=\sqrt{-1},
$$

is satisfied on the hyperplane $(k, \alpha)=0$ for all $\alpha \in R_{+}$. Using the previous methods one can prove the following theorem.

Theorem 2. When such a function $\psi$ exists, it is the eigenfunction of the supercomplete commutative ring, which contains the operator

$$
H_{G}=-\Delta+2 \sum_{\alpha \in R_{+}} \omega^{2}(\alpha, \alpha) \sin ^{-2} \omega(\alpha, x)
$$

and is isomorphic to the ring of all polynomials in $k$ with the property (9).

To construct the eigenfunction $\psi_{m}$ of the operator

$$
H_{G}=-\Delta+m(m+1) \sum_{\alpha \in R_{+}} \omega^{2}(\alpha, \alpha) \sin ^{-2} \omega(\alpha, x)
$$

we demand that $\psi_{m}$ has the same form as in the rational case, but instead of (8) that it must satisfy the relation

$$
\delta_{\omega \alpha}\left[(\alpha, k)^{-1} \delta_{\omega \alpha}\right]^{s} \psi_{m}=0 \quad \text { when } \quad(\alpha, k)=0
$$

for all $\alpha \in R_{+}$and $s=0,1, \ldots, m-1$.

Here by definition

$$
\delta_{\omega \alpha} \Phi(k, x):=\phi(k+i \omega \alpha, x)-\phi(k-i \omega \alpha, x) .
$$

All Weyl group invariant polynomials $P_{1}(k), \ldots, P_{n}(k)$ obviously satisfy (11).

Another example is presented by

$$
P_{n+1}^{(m)}(k)=\prod_{\alpha \in R_{+}}(k, \alpha)\left((k, \alpha)^{2}+\omega^{2}(\alpha, \alpha)\right)\left((k, \alpha)^{2}+4 \omega^{2}(\alpha, \alpha)\right) \ldots\left((k, \alpha)^{2}+m^{2}(\alpha, \alpha)\right) .
$$

It's easy to check that $P_{n+1}^{(m)}(k)$ satisfies the condition (11) and is antiinvariant under the action of the Weyl group. 
To Summarize. In order to prove conjecture 1 in the rational and trigonometric cases it's sufficient to prove the existence of $\psi$ with property (8) or (11). If such a function $\psi$ is given, the commuting operators $L_{i}$ can be found by an effective procedure. In the elliptic case for $m=1$ we conjecture that the variety on which the $\psi$-function "lives" is $M_{G}=\mathbb{C}^{n} / L+\tau L$, which also arises in the generalized Chevalley theorem $[25,26]$ (see Sect. 1). The precise characterization for $\psi$ in this case will be considered in the subsequent paper.

\section{Formulas for the $\psi$-Function of Quantum Calogero Systems}

We now describe a recursive procedure, which leads to formulas for the $\psi$-function in the rational case (3) for the Lie algebra $A_{n-1}: H \psi=-k^{2} \psi$,

$$
H=-\Delta+4 \sum_{i<j}\left(x_{i}-x_{j}\right)^{-2} \quad(i, j=1, \ldots, n),
$$

and prove conjecture 1 in this case. So we consider a Calogero system with special values of the constants in the potential.

For $n=2, \psi$ can easily be found: $\psi=\left[\left(k_{1}-k_{2}\right)-2\left(x_{1}-x_{2}\right)^{-1}\right] \exp (k, x)$. It's easy to check that such a $\psi$-function satisfies condition (6).

We rewrite it in the form

$$
\psi=\left[\left(\partial_{1}-\partial_{2}\right)-2\left(x_{1}-x_{2}\right)^{-1}\right] \exp (k, x)=\mathscr{D}_{2} \exp (k, x),
$$

where $\mathscr{D}_{2}=\partial_{12}-2-12, x_{i j}=x_{i}-x_{j}, \partial_{i}=\partial / \partial x_{i}, \partial_{i j}=\partial_{i}-\partial_{j}$.

For other $n$ the $\psi$-function also has the form

$$
\psi=\mathscr{D}_{n} \exp (k, x),
$$

where the differential operators $\mathscr{D}_{n}$ can be found from the following recurrence formulas:

$$
\mathscr{D}_{n+1}=Q_{n+1}^{1} \ldots^{n} \mathscr{D}_{n} \text {. }
$$

For $Q_{n+1}^{1}{ }^{n}$ there also exist recurrence formulas

$$
\begin{aligned}
Q_{N}^{i_{1} \ldots i_{k}}= & Q_{N}^{i_{1} \ldots i_{k-1}} \partial_{i_{k} N}-2 x_{i_{k} N}^{-1} \Omega_{N}^{i_{1} \ldots i_{k-1}} \\
& +\sum_{s=1}^{k-1} 2 x_{i_{s} i_{k}}^{-2} Q_{N}^{i_{1} \ldots \hat{i}_{s} \ldots i_{k-1},}, Q_{N}=1 .
\end{aligned}
$$

Here $i_{1} \ldots i_{k}$ are different indices less than $N$, the symbol $\hat{i}_{s}$ means that the index $i_{s}$ must be omitted.

In order to show how this procedure works let's find the $\psi$-function for three particles.

According to (17), $\mathscr{D}_{3}=Q_{3}^{12} \mathscr{D}_{2}$, where $\mathscr{D}_{2}$ is determined by (15). From (18)

$$
Q_{3}^{12}=Q_{3}^{1} \partial_{23}-2 x_{23}^{-1} Q_{3}^{1}+2 x_{12}^{-2} Q_{3} \text {. }
$$

Now again using (18),

$$
Q_{3}^{1}=Q_{3} \partial_{13}-2 x_{13}^{-1} Q_{3}=\partial_{13}-2 x_{13}^{-1} \text { because } Q_{3}=1 \text {. }
$$


So we have

$$
\begin{aligned}
Q_{3}^{12} & =\left(\partial_{13}-2 x_{13}^{-1}\right) \partial_{23}-2 x_{23}^{-1}\left(\partial_{13}-2 x_{13}^{-1}\right)+2 x_{12}^{-2} \\
\mathscr{D}_{3} & =\left(\partial_{13} \partial_{23}-2 x_{13}^{-1} \partial_{23}-2 x_{23}^{-1} \partial_{13}+4 x_{23}^{-1} x_{13}^{-1}+2 x_{12}^{-2}\right) \circ\left(\partial_{12}-2 x_{12}^{-1}\right),
\end{aligned}
$$

and after the simple calculations

$$
\begin{aligned}
\mathscr{D}_{3}= & \partial_{12} \partial_{13} \partial_{23}-2 x_{12}^{-1} \partial_{13} \partial_{23}-2 x_{13}^{-1} \partial_{12} \partial_{23}-2 x_{23}^{-1} \partial_{12} \partial_{13} \\
& +4 x_{23}^{-1} x_{13}^{-1} \partial_{12}+4 x_{13}^{-1} x_{12}^{-1} \partial_{23}+4 x_{12}^{-1} x_{23}^{-1} \partial_{13}-12 x_{12} x_{13} x_{23} .
\end{aligned}
$$

Theorem 3. The function $\psi$, determined by the recurrence formulas (16), (17), (18), satisfies the condition (6) and therefore is the eigenfunction of the operator (14): $H \psi=-k^{2} \psi$ and of a supercomplete commutative ring of quantum integrals (14).

Proof. We will use the following two lemmas.

Lemma 1. The operator $Q_{n+1}^{1}{ }^{n}$ is symmetric on the indices $1, \ldots, n$ and has the highest term

$$
\prod_{i=1}^{n}\left(\partial_{i}-\partial_{n+1}\right)
$$

Lemma 2. In order to check the conditions (6) for (16) it's sufficient to prove that the operator $\mathscr{D}_{n}$ is right-divisible by $\partial_{i j} \circ x_{i j}^{-1}$ for all $i<j$.

The last statement of Lemma 1 follows immediately from (18) and ensures the prescribed highest term of $\mathscr{D}_{n+1}$ and $\psi$. The first statement also follows from (18) after some calculations.

To prove Lemma 2 suppose that $\mathscr{D}_{n}$ has a form $\mathscr{D}_{n}=P_{i j} \circ \partial_{i j} \circ x_{i j}^{-1}$ for some differential operator $P_{i j}$. Then

$$
\begin{aligned}
\left(\frac{\partial}{\partial k_{i}}-\frac{\partial}{\partial k_{j}}\right) \psi & =\mathscr{D}_{n}\left(\frac{\partial}{\partial k_{i}}-\frac{\partial}{\partial k_{j}}\right) \exp (k, x)=P_{i j} \circ \partial_{i j} \circ x_{i j}^{-1}\left(x_{i j} \exp (k, x)\right) \\
& =\left(k_{i}-k_{j}\right) P_{i j} \exp (k, x)=0
\end{aligned}
$$

when $k_{i}-k_{j}=0$, i.e. the condition (6) is satisfied.

We will prove the theorem by induction. For $n=2$

$$
\mathscr{D}_{2}=\partial_{12}-2 x_{12}^{-1}=x_{12} \circ \partial_{12} \circ x_{12}^{-1}
$$

and by Lemma $2, \psi$ possess the necessary property. Suppose now that $\mathscr{D}_{n}$ is rightdivisible by $\partial_{i j} \circ x_{i j}^{-1}$, and let us prove that $\mathscr{D}_{n+1}$ also has the analogous properties.

Because of symmetry $Q_{n+1}^{1}{ }^{n}$ (Lemma 1$)$ it is only necessary to show that $\mathscr{D}_{n+1}$ is divisible by $\partial_{n n+1} \circ x_{n n+1}^{-1}$.

Using the relation

$$
\mathscr{D}_{n+1}=Q_{n+1}^{1}{ }^{n} Q_{n}^{1 \ldots n-1} \mathscr{D}_{n-1}
$$

and the commutativity of $\partial_{n n+1} x_{n n+1}^{-1}$ and $\mathscr{D}_{n-1}$, we reduce the problem to the proof that the operator $Q_{n+1}^{1} \dddot{1}^{n} \circ Q_{n}^{1} \ldots n-1 \circ x_{n n+1}$ is right-divisible by $\left(\partial_{n}-\partial_{n+1}\right)$ or in more convenient notation $Q_{N}^{1} \ldots k M \circ Q_{M}^{1} \ldots k \circ x_{M N}$ is right-divisible by $\partial_{M N}$.

The proof of this fact is long with many applications of formulas (18), so we omit some steps. 
First of all we introduce some notation:

$$
\begin{gathered}
Q_{N}=Q_{N}^{1} \ldots k M, \quad Q_{M}=Q_{M}^{1} \cdots k, \quad Q_{M}^{i}=Q_{M}^{1} \ldots \hat{i} \ldots k, \quad Q_{N}^{M}=Q_{N}^{1} \ldots k, \\
Q_{N}^{i}=Q_{N}^{1 \ldots \hat{i} \ldots k M,} \quad Q=Q_{N}^{1} \ldots k M \circ Q_{M}^{1} \ldots k \circ x_{M N} .
\end{gathered}
$$

We have

$$
\begin{aligned}
Q & =Q_{N} \circ Q_{M} \circ x_{M N}=Q_{N} x_{M N} Q_{M}+Q_{N} \circ\left[Q_{M} x_{M N}\right] \\
& =x_{M N} \circ Q_{N} \circ Q_{M}+\left[Q_{N}, x_{M N}\right] Q_{M} \cap Q_{N} \circ\left[Q_{M} x_{M N}\right] .
\end{aligned}
$$

Using (18) one can prove that

$$
\begin{aligned}
& {\left[Q_{M}, x_{M}\right]=-\sum_{i=1}^{k} Q_{M}^{i}, \quad\left[Q_{N}, x_{N}\right]=-\sum_{i=1}^{k} Q_{N}^{i}-Q_{N}^{M},} \\
& {\left[Q_{N}, x_{M}\right]=Q_{N}^{M}, \quad\left[\partial_{M}, Q_{M}\right]=-\sum_{i=1}^{k} 2 x_{i M}^{-2} Q_{M}^{i} .}
\end{aligned}
$$

Substituting this commutator and using (18) one can rewrite the last expression as

$$
\begin{aligned}
Q= & \sum_{i=1}^{k}\left[x_{M N} Q_{N}^{M}\left(-2 x_{i M}^{-2} Q_{M}^{i}\right)+2 x_{M N} x_{i M}^{-2} Q_{N}^{i, M} Q_{M}\right. \\
& +\sum_{j \neq i}\left(Q_{N}^{i, M}\left(-2 x_{j M}^{-2} Q_{M}^{j}\right)+2 x_{j M}^{-2} Q_{N}^{i j M} Q_{M}+2 Q_{N}^{M} x_{j M}^{-2} Q_{M}^{i j}\right) \\
& \left.+2 x_{M N}^{-1}\left(Q_{N}^{M} Q_{M}^{i}-Q_{N}^{i, M} Q_{M}\right)-\sum_{j \neq i} 2 x_{j M}^{-2} Q_{N}^{j, M} Q_{M}^{i}\right] \\
& + \text { terms right-divisible by } \partial_{M N} .
\end{aligned}
$$

In order to prove the theorem it is sufficient to show that after the substitution of $\partial_{i}$ for $\partial_{i M}$ and $\partial_{i N}$ in the last expression the new operator will be equal to zero. Denoting the new expression by $\widetilde{Q}, \widetilde{Q}_{M}, \widetilde{Q}_{N}, \ldots$, we can write the necessary relation as

$$
\begin{aligned}
& \sum_{i=1}^{k}\left[\left(-2 x_{M N} x_{i M}^{-2}+2 x_{M N}^{-1}\right) M_{i}(Q)+\left(4 x_{M N} x_{i M}^{-3}-4 x_{i M}^{-2}\right) Q^{i}\right. \\
& \left.\quad+\sum_{j \neq i}\left(-2 x_{j M}^{-2}\right) \mathscr{L}_{i j}(Q)-4 x_{j M}^{-3} M_{i}\left(Q^{j}\right)\right]=0,
\end{aligned}
$$

where

$$
\begin{gathered}
M_{i}(Q)=\widetilde{Q}_{N}^{M} \widetilde{Q}_{M}^{i}-\widetilde{Q}_{N}^{i M} \widetilde{Q}_{M}{ }^{\prime} \quad Q^{i}=\widetilde{Q}_{N}^{i, M} \widetilde{Q}_{M}^{i}, \\
\mathscr{L}_{i j}(Q)=\tilde{Q}_{N}^{i M} \tilde{Q}_{M}^{j}+\widetilde{Q}_{N}^{j M} \widetilde{Q}_{M}^{i}-\widetilde{Q}_{N}^{i j M} \widetilde{Q}_{M}-\widetilde{Q}_{N}^{M} \widetilde{Q}_{M}^{i j} \quad(i \neq j) .
\end{gathered}
$$

Using (18), one can check the following relations:

$$
\begin{aligned}
M_{i}(Q)= & 2 x_{M N} x_{i M}^{-1} x_{i N}^{-1} Q^{i}-\sum_{j \neq i}\left(4 x_{i j}^{-3} Q^{i j}-2 x_{i j}^{-2} M_{j}\left(Q^{i}\right)\right) \\
\mathscr{L}_{i j}(Q)= & -2 x_{M N} x_{j M}^{-1} x_{j N}^{-1} M_{i}\left(Q^{j}\right) \\
& +\sum_{l \neq i, j}\left[\left(-2 x_{j l}^{-2}\right) \mathscr{L}_{i l}\left(Q^{j}\right)+4 x_{j l}^{-3} M_{i}\left(Q^{j l}\right)\right]-4 x_{i j}^{-2} Q^{i j} .
\end{aligned}
$$


Using this relation we reduce the proof to the following two identities:

$$
\begin{aligned}
& \sum_{\sigma \in S_{k}} x_{\sigma}^{-2} x_{\sigma(k-1) \sigma(k)}^{-1} \equiv 0 \\
& \sum_{\sigma \in S_{k}} x_{\sigma}^{-2}\left(x_{M N}^{2} x_{\sigma(1) M}^{-2} x_{\sigma(k) M}^{-1} x_{\sigma(k) N}^{-1}-x_{\sigma(k) M}^{-1} x_{\sigma(k) N}^{-1}\right. \\
& +x_{M N} x_{\sigma(1) M}^{-2} x_{\sigma(k-1) \sigma(k)}^{-1}-x_{M N} x_{\sigma(1) M}^{-3} x_{\sigma(1) \sigma(2)}^{2} x_{\sigma(k) M}^{-1} x_{\sigma(k) N}^{-1} \\
& +x_{\sigma(1) M}^{-2} x_{\sigma(1) \sigma(2)}^{2} x_{\sigma(1) \sigma(k)}^{-2} \\
& +\sum_{s=1}^{k-1} x_{M N}^{2} x_{\sigma(1) M}^{-2} x_{\sigma(1) \sigma(2)}^{2} x_{\sigma(s) \sigma(s+1)}^{2} x_{\sigma(s) M}^{-1} x_{\sigma(s) N}^{-1} \\
& \times x_{\sigma(1) \sigma(s+1)}^{-2} x_{\sigma(k) M}^{-1} x_{\sigma(k) N}^{-1} \\
& +\sum_{s=1}^{k-1} x_{M N} x_{\sigma(1) M}^{-2} x_{\sigma(1) \sigma(2)}^{2} x_{\sigma(s) \sigma(s+1)}^{2} x_{\sigma(s) M}^{-1} x_{\sigma(s+1) N}^{-1} \\
& \left.\times x_{\sigma(1) \sigma(s+1)}^{-2} x_{\sigma(k-1) \sigma(k)}^{-1}\right) \equiv 0 .
\end{aligned}
$$

Here $\sigma \in S_{k}$ is any permutation of $(1, \ldots, k), x_{\sigma}^{-2}=x_{\sigma(1) \sigma(2)}^{-2} x_{\sigma(2) \sigma(3)}^{-2} \ldots x_{\sigma(k-1) \sigma(k)}^{-2}$. These identities can be proven by calculating the singularities of this expression and using induction.

Theorem 3 is proven.

Remark 1. In the trigonometric case the analogous procedure can be used for finding the $\psi$-function. Instead of relation (18) we write

$$
\begin{aligned}
Q_{N}^{i_{1} \ldots i_{k}}= & Q_{N}^{i_{1} \ldots i_{k-1}} \partial_{i_{k} N}-2 \omega \cot \omega x_{i_{k} N} Q_{N}^{i_{1} \ldots i_{k-1}} \\
& +\sum_{s=1}^{k-1} 2 \omega^{2} \sin ^{-2} \omega x_{i_{s} i_{k}} Q_{N}^{i_{1} \ldots \hat{i}_{s} \ldots i_{k-1}}, \quad Q_{N}=1 .
\end{aligned}
$$

We conjecture that the corresponding function $\psi$ will satisfy the condition (9) and therefore must be an eigenfunction of the Sutherland operator,

$$
H=-\Delta+4 \omega^{2} \sum_{i<j} \sin ^{-2} \omega\left(x_{i}-x_{j}\right)
$$

$H \psi=-k^{2} \psi$, but we do not have the complete proof of this fact. Of course the same remark concerns also the hyperbolic case.

Remark 2. For $g_{\alpha}=2(\alpha, \alpha)$ and $G=A_{n}$ the operators (2), (3) are equivalent to the radial parts of the Laplace-Beltrami operators on symmetric spaces of type AII in Cartan's notation [17]. So our results can be interpreted in terms of zonal spherical functions, for which only integral representations are known. Notice that there exists only one more such case: when $g_{\alpha}=12$ and $G=A_{2}$ the operators (2), (3) are equivalent to the radial part of the Laplace-Beltrami operator on symmetric space of type EIV. For all other symmetric spaces the corresponding $g_{\alpha}$ do not have the form $m(m+1)(\alpha, \alpha)$ for a natural number $m$ (see [17]). 


\section{Examples: $\psi$-Functions for the Lie Algebras of Rank 1 and 2}

We begin with the case of $G=A_{1}$.

Example 1. In this case we have

$$
H=-\left(\partial_{1}^{2}+\partial_{2}^{2}\right)+2 m(m+1) \mathscr{P}\left(x_{1}-x_{2}\right) .
$$

Introducing the new variable $y=x_{1}-x_{2}$ one can reduce the problem to the one-dimensional

$$
\hat{H}=-\frac{\partial^{2}}{\partial y^{2}}+m(m+1) \mathscr{P}(y)
$$

It is the well-known Lamé operator and it was shown by Ince that $\hat{H}$ has in its spectrum exactly $m$ gaps. The eigenfunction $\psi$ can be expressed by means of elliptic $\sigma$-functions.

In the trigonometric case

$$
\hat{H}=-\partial^{2} / \partial y^{2}+m(m+1) \omega^{2} \sin ^{-2} \omega y,
$$

and the $\psi$-function can be presented in the form

$\psi=(\partial-m \omega \cot \omega y)(\partial-(m-1) \omega \cot \omega y) \ldots(\partial-\omega \cot \omega y) \exp (k, y), \quad \partial=\partial / \partial y$.

For example, for $m=1$,

$$
\psi=(k-\omega \cot \omega y) \exp (k y) .
$$

In the rational case

$$
\begin{gathered}
\hat{H}=-\partial^{2}+m(m+1) y^{-2}, \quad \hat{H} \psi=-k^{2} \psi, \\
\psi=\left(\partial-m y^{-1}\right)\left(\partial-(m-1) y^{-1}\right) \ldots\left(\partial-y^{-1}\right) \exp (k y) .
\end{gathered}
$$

In all these cases there exists a commuting operator $A:[\hat{H}, A]=0$ of order $2 m+1$, which can be found by a general procedure (see for example [2-4]). For instance when $m=1$,

$$
A=-\partial^{3}+3 f(y) \partial+\frac{3}{2} f^{\prime}(y), \quad \text { where } f=\mathscr{P}(y), \quad \sin ^{-2} \omega y \text { or } y^{-2} .
$$

Example 2. $G=A_{2}$ : Sutherland operator for three particles $(m=1)$,

$$
H=-\left(\partial_{1}^{2}+\partial_{2}^{2}+\partial_{3}^{2}\right)+4 \sum_{1 \leqq i<j \leqq 3} \sin ^{-2} \omega\left(x_{i}-x_{j}\right) .
$$

It can be checked that the function $\psi$ is given by

$$
\begin{aligned}
\psi= & \left(k_{12} k_{13} k_{23}-2 \omega \cot \omega x_{12} k_{13} k_{23}-\ldots\right. \\
& +4 \omega^{2} \cot \omega x_{12} \cot \omega x_{13} k_{23}+\ldots \\
& -8 \omega^{3} \cot \omega x_{12} \cot \omega x_{12} \cot \omega x_{23} \\
& \left.-4 \omega^{3} \sin ^{-1} \omega x_{12} \sin ^{-1} \omega x_{13} \sin ^{-1} \omega x_{23}\right) \\
& \times \exp \left(k_{1} x_{1}+k_{2} x_{2}+k_{3} x_{3}\right),
\end{aligned}
$$


(where we omit the terms which differ only by permutation of indices), and satisfies condition (9) and $H \psi=-k^{2} \psi$.

As a limiting case we have the formula (16), (19) for the $\psi$-function for the corresponding Calogero system with three particles.

We conjecture that the $\psi$-function for $m>1$ can be presented in a factorized form as in example 1 (see (25)), where instead of $\left(\partial-S x^{-1}\right)$ one has to use a modification of the operator $\mathscr{D}_{3}$ (19). The same is possibly true in the trigonometric case (24), (26).

Example 3. $G=B_{2} \approx C_{2}$.

$$
\begin{gathered}
H=-\left(\partial_{1}^{2}+\partial_{2}^{2}\right)+2 \omega^{2} \sin ^{-2} \omega x_{1}+2 \omega^{2} \sin ^{-2} \omega x_{2} \\
+4 \omega^{2} \sin ^{-2} \omega\left(x_{1}+x_{2}\right)+4 \omega^{2} \sin ^{-2} \omega\left(x_{1}-x_{2}\right) \\
H \psi=-k^{2} \psi, \\
\psi=\left[\left(\partial_{1}-\partial_{2}-2 \omega \cot \omega\left(x_{1}-x_{2}\right)\right)\left(\partial_{1}+\partial_{2}-2 \omega \cot \omega\left(x_{1}+x_{2}\right)\right)\right. \\
\left.-2 \omega^{2} \sin ^{-2} \omega x_{1}+2 \omega^{2} \sin ^{-2} \omega x_{2}\right]\left(\partial_{1}-\omega \cot \omega x_{1}\right)\left(\partial_{2}-\omega \cot \omega x_{2}\right) \\
\times \exp \left(k_{1} x_{1}+k_{2} x_{2}\right),
\end{gathered}
$$

or in more explicit form,

$$
\begin{aligned}
\psi= & {\left[\left(k_{1}-k_{2}\right)\left(k_{1}+k_{2}\right) k_{1} k_{2}-\omega \cot \omega x_{1} k_{2}\left(k_{1}+k_{2}\right)\left(k_{1}-k_{2}\right)\right.} \\
& -\omega \cot \omega x_{2} k_{1}\left(k_{1}+k_{2}\right)\left(k_{1}-k_{2}\right)-2 \omega \cot \omega\left(x_{1}+x_{2}\right) k_{1} k_{2}\left(k_{1}-k_{2}\right) \\
& -2 \omega^{2} \cot \omega\left(x_{1}-x_{2}\right) k_{1} k_{2}\left(k_{1}+k_{2}\right)+\omega^{2} \cot \omega x_{1} \cot \omega x_{2}\left(k_{1}+k_{2}\right)\left(k_{1}-k_{2}\right) \\
& +2 \omega \cot \omega x_{1} \cot \omega\left(x_{1}+x_{2}\right) k_{2}\left(k_{1}-k_{2}\right)+2 \omega^{2} \cot \omega x_{2} \cot \omega\left(x_{1}+x_{2}\right) \\
& \times k_{1}\left(k_{1}-k_{2}\right)+2 \omega^{2} \cot \omega x_{2} \cot \omega\left(x_{1}-x_{2}\right) k_{1}\left(k_{1}+k_{2}\right) \\
& +4 \omega^{2} \cot \omega\left(x_{1}-x_{2}\right) \cot \omega\left(x_{1}+x_{2}\right) k_{1} k_{2}-2 \omega^{3} \cot \omega x_{1} \cot \omega x_{2} \\
& \times \cot \omega\left(x_{1}+x_{2}\right)\left(k_{1}-k_{2}\right)-2 \omega^{3} \cot \omega x_{1} \cot \omega x_{2} \cot \omega\left(x_{1}-x_{2}\right)\left(k_{1}+k_{2}\right) \\
& -4 \omega^{3} \cot \omega x_{1} \cot \omega\left(x_{1}-x_{2}\right) \cot \omega\left(x_{1}+x_{2}\right) k_{2}-4 \omega^{3} \cot \omega x_{2} \\
& \times \cot \omega\left(x_{1}-x_{2}\right) \cot \omega\left(x_{1}+x_{2}\right) k_{1} \\
& +4 \omega^{4} \cot \omega x_{1} \cot \omega x_{2} \cot \omega\left(x_{1}-x_{1}\right) \cot \omega\left(x_{1}+x_{2}\right) \\
& \left.+8 \omega^{4} \cot \omega x_{1} \cot \omega x_{2} \sin ^{-1} \omega\left(x_{1}+x_{2}\right) \sin ^{-1} \omega\left(x_{1}-x_{2}\right)\right] \exp \left(k_{1} x_{1}+k_{2} x_{2}\right) .
\end{aligned}
$$

A formula for $\psi$ in rational case

$$
H=-\left(\partial_{1}^{2}+\partial_{2}^{2}\right)+2 x_{1}^{-2}+2 x_{2}^{-2}+4\left(x_{1}-x_{2}\right)^{-2}+4\left(x_{1}+x_{2}\right)^{-2}
$$

can be found from the previous one in the limit $\omega \rightarrow 0$. Changing $\omega \rightarrow i \omega$ we come to the hyperbolic case $\left(2^{\prime}\right)$.

\section{Example 4. $G=G_{2}$}

The root system is shown in Fig. 1 . We realize it in the plane in $\mathbb{R}^{3}$, so that

$$
\begin{gathered}
(\alpha, x)=x_{1}-x_{2}, \quad(\beta, x)=-x_{1}+2 x_{2}-x_{3}, \quad(\alpha+\beta, x)=x_{2}-x_{3}, \\
(3 \alpha+2 \beta, x)=x_{1}+x_{2}-2 x_{3}, \quad(2 \alpha+\beta, x)=x_{1}-x_{3}, \quad(3 \alpha+\beta, x)=2 x_{1}-x_{3}
\end{gathered}
$$




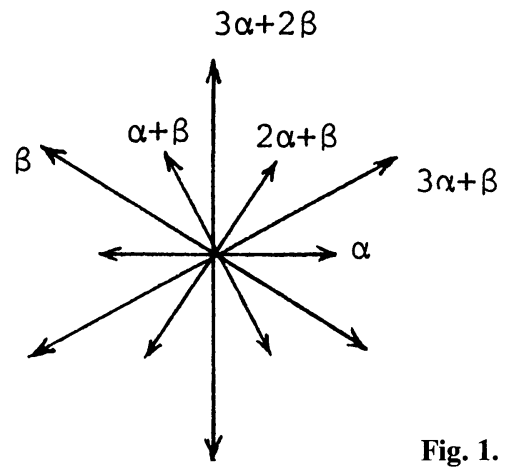

and

$$
\begin{aligned}
H=-\left(\partial_{1}^{2}+\partial_{2}^{2}+\partial_{3}^{2}\right) & +\frac{4}{\left(x_{1}-x_{2}\right)^{2}}+\frac{4}{\left(x_{1}-x_{3}\right)^{2}}+\frac{4}{\left(x_{2}-x_{3}\right)^{2}}+\frac{12}{\left(x_{1}-2 x_{2}+x_{3}\right)^{2}} \\
& +\frac{12}{\left(x_{1}+x_{2}-2 x_{3}\right)^{2}}+\frac{12}{\left(2 x_{1}-x_{2}-x_{3}\right)^{2}} .
\end{aligned}
$$

The root system $G_{2}$ consists of two systems of type $A_{2}: G_{2}=A_{2} \cup A_{2}^{\prime}$. We introduce the corresponding operators $\mathscr{D}_{A_{2}}$ and $\mathscr{D}_{A^{\prime}}$, where $\mathscr{D}_{A_{2}}$ is determined by (19) and

$$
\begin{aligned}
\mathscr{D}_{A_{2}}^{\prime}=\partial_{\beta} \partial_{3 \alpha+2 \beta} \partial_{3 \alpha+\beta} & -6(\beta, x)^{-1} \partial_{3 \alpha+\beta} \partial_{3 \alpha+2 \beta}-\ldots \\
+ & 36(\beta, x)^{-1}(3 \alpha+\beta, x)^{-1} \partial_{3 \alpha+2 \beta}+\ldots \\
& -12 \cdot 3^{3}(\beta, x)^{-1}(3 \alpha+\beta, x)^{-1}(3 \alpha+2 \beta, x)^{-1} .
\end{aligned}
$$

Now we can write the formula for $\psi$ :

$$
\begin{aligned}
\psi= & {\left[\left(\mathscr{D}_{A_{2}}^{\prime}+18(\alpha, x)^{-2} \partial_{3 \alpha+2 \beta}-18(2 \alpha+\beta, x)^{-2} \partial_{\beta}\right.\right.} \\
& -18(\alpha+\beta, x)^{-2} \partial_{3 \alpha+\beta}+108(\beta, x)^{-1}(2 \alpha+\beta, x)^{-2} \\
& +108(3 \alpha+\beta, x)^{-1}(\alpha+\beta, x)^{-2}-108(3 \alpha+2 \beta, x)^{-1}(\alpha, x)^{-2} \\
& \left.\left.-12 \cdot 3^{4}(\beta, x)^{-1}(3 \alpha+\beta, x)^{-1}(3 \alpha+2 \beta, x)^{-1}\right) \times \mathscr{D}_{A_{2}}\right] \\
& \times \exp \left(k_{1} x_{1}+k_{2} x_{2}+k_{3} x_{3}\right) .
\end{aligned}
$$

In the trigonometric case the analogous formula can be written.

Remark. It can be shown that the operators in all these examples are "finite gap" in every energy level in the sense of $[7,8]$. The corresponding curves are singular and the divisor $\mathscr{D}$ is $N\left(P_{1}+P_{2}\right)$, where $P_{1}$ and $P_{2}$ are "the infinities" (see [7,8], $N$ is the number of positive roots.

Acknowledgements. We would like to thank I. M. Krichever for very useful discussions about the analytic properties of the $\psi$-function. One of the authors (A.P.V.) thanks Prof. J. Moser for the kind hospitality at the Forschungsinstitut für Mathematik and H. Knörrer and E. Trubowitz for stimulating discussions. 
Note added in the proof. Recently H. Knörrer showed one of the authors (A.P.V.) interesting papers $[29,30]$, in which some results for the quantum problem (2) and (3) with general coefficients $g_{\alpha}$ are found. The problem of supercomplete commutative rings is not discussed in these papers. It would be useful to compare our methods with those of [29, 30]; possibly it will help to prove conjecture 1 .

\section{References}

1. Burchnal, J.L., Chaundy, T.W.: Commutative ordinary differential operators. I, II. Proc. Lond. Math. Soc. 21, 420-440 (1922); Proc. Royal Soc. Lond. 118, 557-583 (1928)

2. Novikov, S.P.: Periodic problem for Korteweg-de Vries equation. I. Funct. Anal. i ego Pril. 8, 54-66 (1974)

3. Lax, P.: Periodic solution of the KdV equation. Commun. Pure Appl. Math. 28, 141-188 (1975)

4. Dubrovin, B.A., Matveev, V.B., Novikov, S.P.: Nonlinear equations of Korteweg-de Vries type, finite-zone linear operators and abelian varieties. Russ. Math. Surv. 31, 51-125 (1976)

5. Krichever, I. M.: Commutative rings of ordinary linear differential operators. Funct. Anal. i ego Pril. 12, 12-31 (1978)

6. Grinevich, P.G.: Ph. D. Thesis, Moscow Univ., 1985

7. Dubrovin, B.A., Krichever, I.M., Novikov, S.P.: Schrödinger equation in magnetic field and Riemann surfaces. Sov. Math. Dokl. 17, 947-951 (1976)

8. Novikov, S.P., Veselov, A.P.: Two-dimensional Schrödinger operator: inverse scattering transform and evolutional equations. Physica 18D, 267-273 (1986)

9. Krichever, I.M.: Methods of algebraic geometry in the theory of nonlinear equations. Usp. Mat. Nauk 32, 198 (1977). English transl. in Russ. Math. Surv. 32, • (1977)

10. Bourbaki, N.: Groupes et algebres de Lie, Chaps. 4-6. Paris: Hermann 1968

11. Calogero, F.: Solution of the one-dimensional $n$-body problem with quadratic and/or inversely quadratic pair potentials. J. Math. Phys. 12, 419-436 (1971)

12. Sutherland, B.: Exact results for a quantum many-body problem in one-dimension. Phys. Rev. A4, 2019-2021 (1971); Phys. Rev. A5, 1372-1376 (1972)

13. Moser, J.: Three integrable Hamiltonian systems, connected with isospectral deformations. Adv. Math. 16, 1-23 (1975)

14. Calogero, F.: Exactly solvable one-dimensional many-body problems. Lett. Nuovo Cimento 13, 411-416 (1975)

15. Olshanetsky, M.A., Perelomov A.M.: Completely integrable Hamiltonian systems associated with semisimple Lie algebras. Inv. Math. 37, 93-108 (1976)

16. Olshanetsky, M.A., Perelomov, A.M.: Quantum completely integrable systems connected with semisimple Lie algebras. Lett. Math. Phys. 2, 7-13 (1977)

17. Olshanetsky, M.A., Perelomov, A.M.: Quantum integrable systems related to Lie algebras. Phys. Rep. 94, 313-404 (1983)

18. Veselov, A.P.: Integrable polynomial mappings and Lie algebras. In the book: "Geometry, differential equations and mechanics." Izdat. Moscow Univ., Moscow, 1986 (Russian)

19. Veselov, A.P.: Integrable mappings and Lie algebras. Sov. Math. Dokl. 35, 211-213 (1987)

20. Julia, G.: Memoire sur la permutabilité des fraction rationelles. Ann. Sci. Ecole Norm. Sup. 39, 131-215 (1922)

21. Fatou, P.: Sur l'iteration analytique et les substitution permutables. J. Math. Pure Appl. 3, 1-49 (1924)

22. Ritt, J.: Permutable rational functions. Trans. Am. Math. Soc. 25, 339-448 (1923)

23. Veselov, A.P.: The dynamics of mappings of toric varieties connected with Lie algebras. Proc. of Top. Conf. (Baku 1987)

24. Chalykh, O.A.: On some properties of polynomial mappings connected with Lie Algebras. Vestnik Mosc. Univ. 3, 57-59 (1988) 
25. Looijenga, E.: Root systems and elliptic curves. Invent. Math. 38, 17-32 (1976)

26. Bernstein, I.N., Schwarzman, O.V.: Chevalley theorem for the complex crystallographical Coxeter groups. Funct. Anal. i Prilozen 12, 79-80 (1978)

27. Bogoyavlensky, O.I.: On perturbations of the Toda lattice. Commun. Math. Phys. 51, 201209 (1976)

28. Veselov, A.P., Chalykh, O.A.: Quantum Calogero problem and commutative rings of manydimensional differential operators. Usp. Mat. Nauk 43, 173 (1988) (Russian)

29. Heckman, G.J., Opdam, E. M.: Root systems and hypergeometric functions. I. Comp. Math. 64, 329-352 (1987)

30. Heckman, G.J.: Root systems and hypergeometric functions. II. Comp. Math. 64, 353-373 (1987)

Communicated by A. Jaffe

Received March 10, 1989 
